\title{
Uncertainty evaluation of nuclear reaction model parameters using integral and microscopic measurements. Covariances evaluation with CONRAD code
}

\author{
C. De Saint Jean ${ }^{\text {a }, ~ B . ~ H a b e r t, ~ P . ~ A r c h i e r, ~ G . ~ N o g u e r e, ~ D . ~ B e r n a r d, ~ J . ~ T o m m a s i, ~ a n d ~ P . ~ B l a i s e ~}$
}

CEA, DEN, Cadarache, 13108 Saint Paul-les-Durance, France

\begin{abstract}
In the $[\mathrm{eV} ; \mathrm{MeV}]$ energy range, modelling of the neutron induced reactions are based on nuclear reaction models having parameters. Estimation of co-variances on cross sections or on nuclear reaction model parameters is a recurrent puzzle in nuclear data evaluation. Major breakthroughs were asked by nuclear reactor physicists to assess proper uncertainties to be used in applications. In this paper, mathematical methods developped in the CONRAD code[2] will be presented to explain the treatment of all type of uncertainties, including experimental ones (statistical and systematic) and propagate them to nuclear reaction model parameters or cross sections. Marginalization procedure will thus be exposed using analytical or Monte-Carlo solutions. Furthermore, one major drawback found by reactor physicist is the fact that integral or analytical experiments (reactor mock-up or simple integral experiment, e.g. ICSBEP, ...) were not taken into account sufficiently soon in the evaluation process to remove discrepancies. In this paper, we will describe a mathematical framework to take into account properly this kind of information.
\end{abstract}

\section{Introduction}

Evaluating uncertainties and correlations in the nuclear data field is of great interest for reactors physicists as it is a major contribution in their own uncertainty propagation evaluation [1].

In this paper, mathematical methods will be presented to explain how to treat all type of uncertainties (including experimental ones, statistical and systematic ones) and propagate them to nuclear reaction model parameters or cross sections.

In addition, as one major drawback found by reactor physicist is the fact that most of the time the integral (or analytical) experiments were not taken into account sufficiently soon in the evaluation process to remove discrepancies, we will present the methodology used in the CONRAD code [2] for a direct use of these integral experiment in the nuclear data models.

\section{Marginalization: Proper estimation of Covariances}

Model Parameter Definition

Cross section are based on nuclear reaction models with parameters not always predicted by theory. In this paper, model parameters will be referred as $\mathbf{x}$. In our energy domain, they can be $E_{\lambda}, \gamma_{\lambda}^{i}, R_{e f f}, \ldots$ in the resolved resonance range, $D_{0},\left\langle\Gamma^{i}\right\rangle, R_{e f f}, \ldots$, in the unresolved resonance range or optical model parameters for higher energies. These parameters are the vectors of uncertainties and correlations because they are used to calculate cross sections covariances.

\footnotetext{
a e-mail: cyrille.de-saint-jean@cea.fr
}

This is an Open Access article distributed under the terms of the Creative Commons Attribution-Noncommercial License 3.0, which permits unrestricted use, distribution, and reproduction in any noncommercial medium, provided the original work is properly cited. 

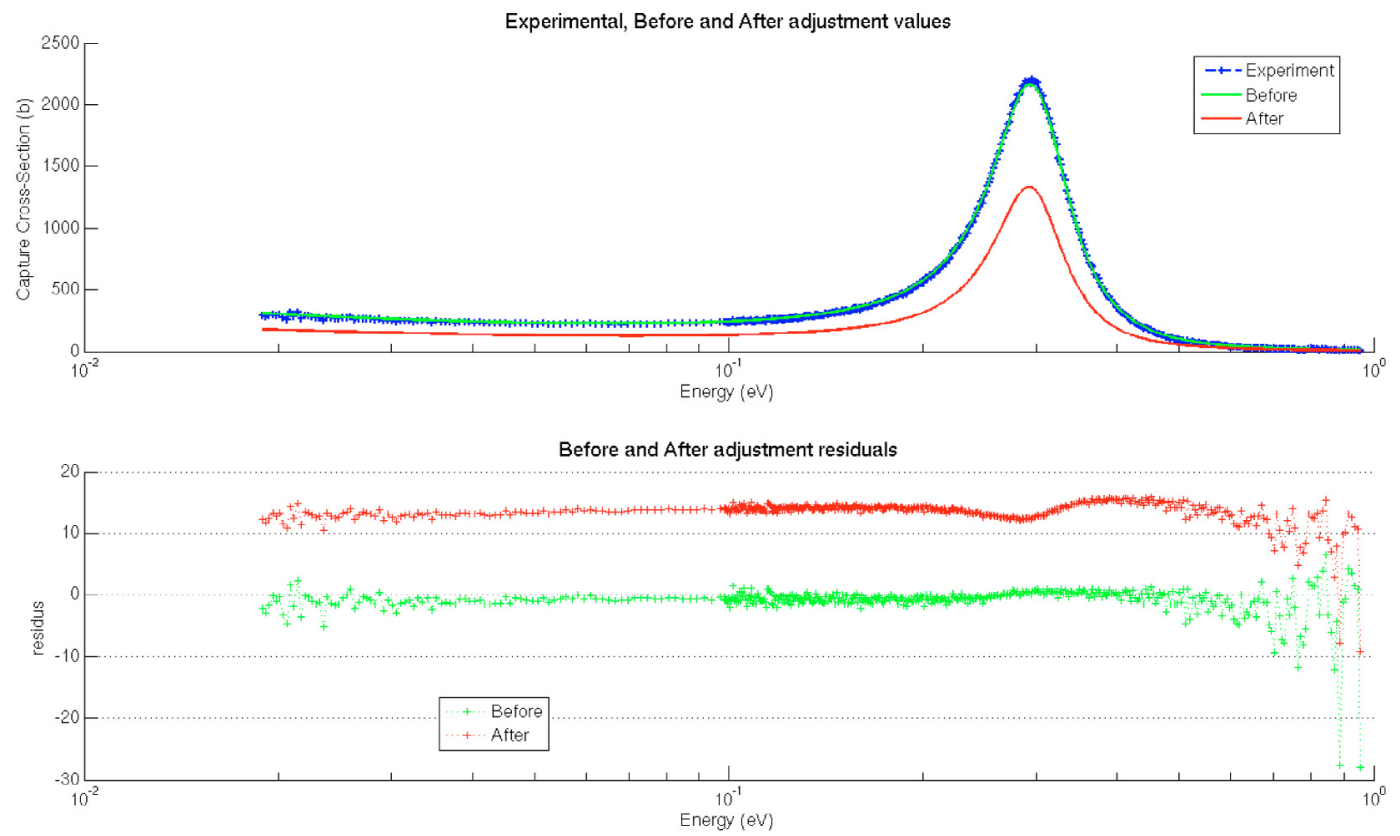

Fig. 1. ${ }^{239} \mathrm{Pu}$ capture cross section (before and after adjustment) and residuals.

Nuisance Parameter Definition

During the data assimilation process, experimental parameters (sample composition, the sample temperature, the energy resolution of the facility, the normalization factor and the background corrections, ... ) play a major role on the uncertainty propagation. In parameter estimation techniques, these additional ingredients are often called nuisance variables whose properties are not of direct interest in itself, but are fundamental for assessing reliable model parameters. Nuisance parameters will be referred as $\theta$ in this paper.

Peele's pertinent puzzle

A first solution to treat experimental nuisance parameters is to re-analyse experiments from raw data with a proper systematic uncertainty description. One will thus obtain a full experimental covariance matrix.

Then, a puzzling effect of the systematic uncertainties (known as the Peele Pertinent Puzzle) will occur. Peele noticed that the minimization of the a least square function could lead to abnormally low results.

We were confronted to this problem by adjusting the $1^{\text {st } 239} \mathrm{Pu}$ resonance on the Gwin capture measurement made in 1971 [3]. The data are used until $1 \mathrm{eV}$. There is no uncertainty in the base EXFOR, we chose to associate with this measure a $1 \%$ statistical uncertainty and $3 \%$ of systematic uncertainty on normalisation.

The adjusted parameters are the energy and the radiative and neutronic widths. Results are given in figure 1 for capture cross section before and after adjustment: the adjusted effective section beeing smaller.

To treat properly the effect of such systematic uncertainties, we thus developped in CONRAD, marginalization techniques.

\subsection{Marginalization}

\subsubsection{Basics of parameter estimation with Bayes' theorem}

Let $\mathbf{y}=\overrightarrow{y_{i}}\left(i=1 \ldots N_{y}\right)$ denote some experimentally measured variables, and let $\mathbf{x}$ denote the parameters defining the model used to simulate theoretically these variables and $\mathbf{t}$ the associated calculated values to be compared with $\mathbf{y}$. 
Using Bayes' theorem [4] and especially its generalisation to continuous variables [5], one can settle the following relation between conditional probability density functions (written $\mathrm{p}($.$) ) when the$ analysis of a new data set $\mathbf{y}$ is performed:

$$
p(\mathbf{x} \mid \mathbf{y}, U)=\frac{p(\mathbf{x} \mid U) \cdot p(\mathbf{y} \mid \mathbf{x}, U)}{\int d \mathbf{x} \cdot p(\mathbf{x} \mid U) \cdot p(\mathbf{y} \mid \mathbf{x}, U)}
$$

where U represents the "background" or "prior" information from which the prior knowledge of $\mathbf{x}$ is assumed. $\mathrm{U}$ is supposed independent of $\mathbf{y}$. In this framework, the denominator is just a normalization constant. To solve this problem, one has to make some assumptions on the prior probability distribution involved. Given a covariance matrix and mean values, the choice of a multivariate joint normal for the probability density $p(\mathbf{x} \mid U)$ is maximizing the entropy [6]. After an additional approximation (so-called Laplace approximation [7]), if the expectation and covariance matrix are $\mathbf{x}_{m}$ and $M_{x}$, the evaluation of posterior expectation and covariances are done by finding the minimum of the following cost function (a generalized least-square):

$$
\chi_{G L S}^{2}=\left(\mathbf{x}-\mathbf{x}_{m}\right)^{T} M_{x}^{-1}\left(\mathbf{x}-\mathbf{x}_{m}\right)+(\mathbf{y}-\mathbf{t})^{T} M_{y}^{-1}(\mathbf{y}-\mathbf{t}) .
$$

Numerical iterative solutions

By using a Gauss-Newton iterative solution, one may find (after some linear algebra):

$$
\begin{aligned}
& \mathbf{x}^{(n)}=\mathbf{x}^{(n-1)}-M_{x}^{(n)} \cdot\left[G^{(n-1)^{T}} M_{y}^{-1}\left(\mathbf{t}\left(\mathbf{x}^{(n-1)}\right)-\mathbf{y}\right)+M_{x}^{\text {prior }^{-1}}\left(\mathbf{x}^{(n-1)}-\mathbf{x}_{\text {prior }}\right)\right] \\
& M_{x}^{(n)}=\left[M_{x}^{\text {prior }}-1+G^{(n-1)^{T}} M_{y}^{-1} G^{(n-1)}\right]^{-1} .
\end{aligned}
$$

Where $G$ represents the matrix of derivatives:

$$
(G)_{i j}=\left(\frac{\partial t_{i}}{\partial x_{j}}\right)
$$

with $t^{i}$ is the theoretical modelization of $\mathrm{i}^{\text {th }}$ experimental value.

In CONRAD, we choose to stop the iteration process when the value of $\left(\chi_{G L S}^{2}\right)^{(n)}$ is converged.

\subsubsection{Marginalization techniques}

With nuisance parameters, the problem is then to evaluate the influence of $\theta$ parameters during the estimation of $\mathbf{x}$ parameters. The use of Bayesian marginalization of the $\theta$ parameters [9] can be used to treat properly nuisance parameters:

$$
p_{\theta}(\mathbf{x} \mid \mathbf{y}, U)=\int d \theta \cdot p(\theta \mid U) \cdot \frac{p(\mathbf{x} \mid U) \cdot p(\mathbf{y} \mid \mathbf{x}, \theta, U)}{\int d \mathbf{x} \cdot p(\mathbf{x} \mid U) \cdot p(\mathbf{y} \mid \mathbf{x}, \theta, U)} .
$$

The marginalization is then the estimation of the first two moments of $p_{\theta}(\mathbf{x} \mid \mathbf{y}, U)$

Two methods are implemented in CONRAD : a Monte-Carlo propagation calculating the first two moment of marginalized distribution [9] or an Analytical solution working with additional hypothesis on probability density function (gaussians) [10].

In figure 2, are presented results of marginalisation in the resolved resonance region for ${ }^{23} \mathrm{Na}[12]$.

We see that after a proper estimation of uncertainties, we may end up with fairly high values (around 3-6 percents in the exmaples). Additional information is necessary in these region to lower these uncertainties. We propose to use clean integral experiments. 

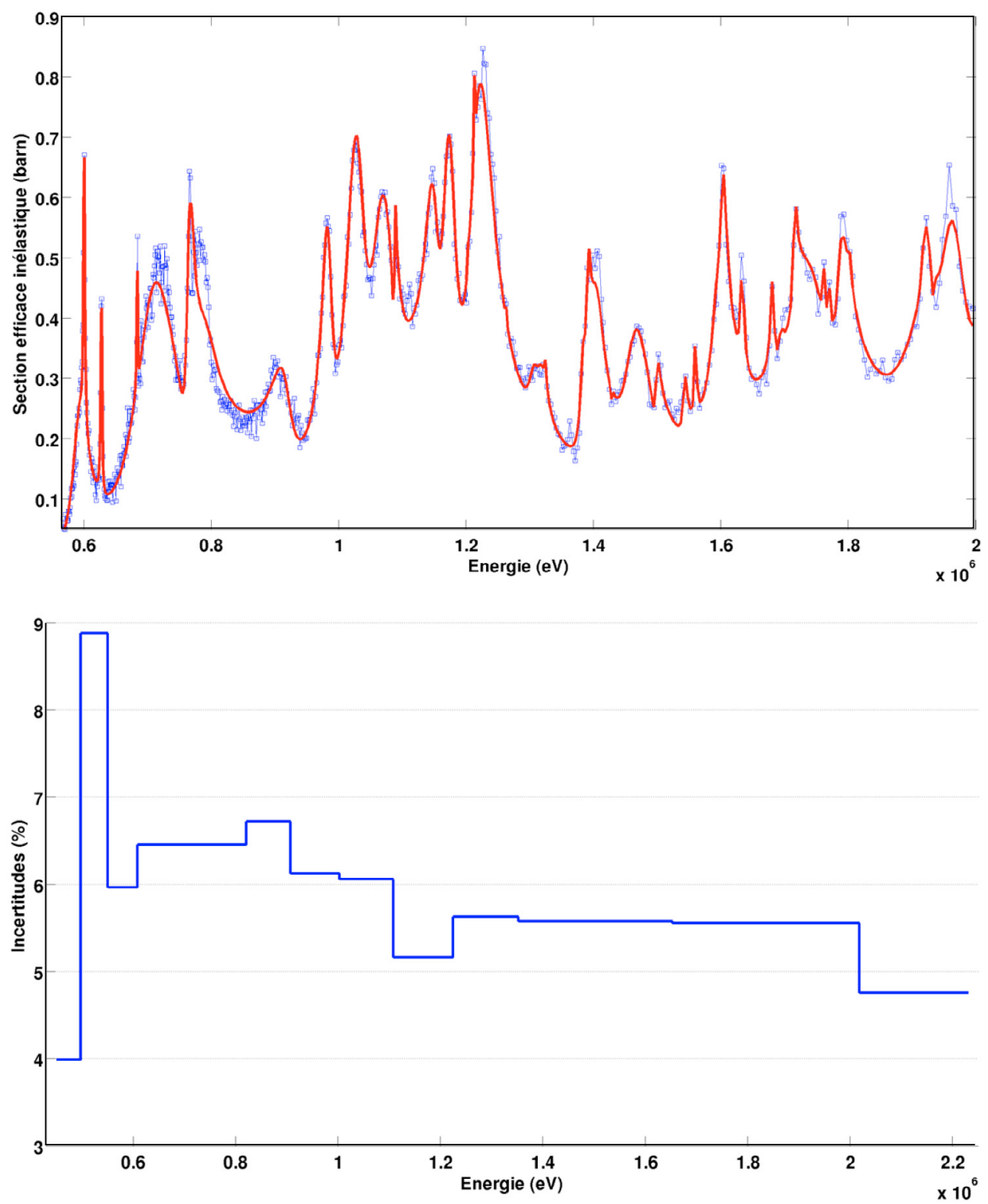

Fig. 2. ${ }^{23} \mathrm{Na}$ inelastic cross section with $6 \%$ uncertainties on normalization.

\section{Integral experiments}

One major drawback found by reactor physicist is the fact that most of the time the integral (or analytical) experiments were not taken into account sufficiently soon in the evaluation process to remove discrepancies.

In the 70's and 80's, in particular at CEA-Cadarache, physicists decided to use integral experiments to assess multigroup cross sections uncertainties by using adjustment procedure [11]. We will first remind this methodology in a similar mathematical framework and then present a new methodology consisting in using integral dedicated experiments directly in the nuclear reaction model evaluation process. Thus, in this paper, to be considered as a good candidate for nuclear data estimation, these experiments must be well described experiment (C/E discrepancies targeted), properly calculated (bias calculated (C/C') and sensitivity coefficients available). 


\subsection{Traditional Multigroup cross section adjustment}

In this section, the $\mathbf{x}$ parameters are indeed the multigroup cross sections themselves.

With the following change in notations :

$-\mathbf{x} \rightarrow \sigma$ : vector of size $\mathrm{N}_{\sigma}=$ Number of Isotopes $\times$ Number of Reactions $\times$ Number of energy groups

- $M_{\sigma}$ is the a priori covariance matrix on multigroup cross sections.

$-\mathbf{y} \rightarrow \mathbf{E}$ : vector of size $\mathrm{N}_{E}=$ Number of Integral Experiments

- $M_{E}$ is then experimental covariance matrix

$-\mathbf{t} \rightarrow \mathbf{C}$ : vector of size $\mathrm{N}_{E}=$ Number of Integral Experiments

$\mathbf{E}$ is a set of measurements which is related to cross sections $\left(\mathrm{k}_{e f f}, \ldots\right)$ and $\mathbf{C}$ its associated set of calculated values. Then, generalized least-square may be written as follows:

$$
\chi_{G L S}^{2}=\left(\sigma-\sigma_{m}\right)^{T} M_{\sigma}^{-1}\left(\sigma-\sigma_{m}\right)+(\mathbf{E}-\mathbf{C}(\sigma))^{T} M_{E}^{-1}(\mathbf{E}-\mathbf{C}(\sigma))
$$

\subsubsection{Information related to integral experiments simulation}

Using a first order approximation, one can write:

$$
\mathbf{C}(\sigma)=\mathbf{C}\left(\sigma_{m}\right)+S \cdot\left(\sigma-\sigma_{\mathbf{m}}\right)
$$

$\mathrm{S}$ is a matrix (size $\mathrm{N}_{E} \times \mathrm{N}_{\sigma}$ ) of calculated derivatives supposed to be constant (when the cross-sections slighly change):

$$
S_{i j}=\frac{\partial C_{i}}{\partial \sigma_{j}}
$$

Most of the time, $\mathrm{S}$ is referred to sensitivities:

$$
S_{i j}=\frac{\partial C_{i}}{\partial \sigma_{j}} \cdot \frac{\sigma_{j}}{C_{i}} .
$$

\subsection{New Methodology: Nuclear data evaluation}

\subsubsection{Mathematicals solutions with Integral measurements}

We keep the notation $\mathbf{E}$ and $M_{E}$ used in the previous section for integral experiments. The associated theoretical values $\mathbf{C}$, is depending on $\mathbf{x}$ the following way:

$$
\mathbf{C}(\sigma(\mathbf{x}))
$$

where $\mathbf{x}$ is now the nuclear reaction model parameter set.

The solution is to mix both microscopic and integral measurements in the same generalized least square:

$$
\begin{aligned}
\chi_{G L S}^{2}= & \left(\mathbf{x}-\mathbf{x}_{m}\right)^{T} M_{x}^{-1}\left(\mathbf{x}-\mathbf{x}_{m}\right)+(\mathbf{y}-\mathbf{t})^{T} M_{y}^{-1}(\mathbf{y}-\mathbf{t})+(\mathbf{E}-\mathbf{C}(\sigma(\mathbf{x})))^{T} M_{E}^{-1} \\
& \times(\mathbf{E}-\mathbf{C}(\sigma(\mathbf{x})))
\end{aligned}
$$

$\mathbf{y}$ beeing the set of microscopic experiments and $\mathbf{E}$ the set of integral experiments.

To implement previous equations in CONRAD [2] (integral experiment in the cost function), one has to calculate the gradient of $\mathbf{C}$ with respect to the $\mathbf{x}$ parameter set:

$$
G(i, j)=\frac{\partial C_{i}}{\partial x_{j}}
$$




\subsubsection{Best practice to take into account integral experiments}

The participation of the integral experiment in the cost function can be far lower than those of microscopic experiments. Numerical problems can arise (round offs, un-sensitive parameters). Treating both type together may therefore in some analyses hide the integral effects.

One has to make some assumptions on the level of knowledge associated to the prior evaluation.

Suppose that, for a given isotope ${ }_{Z}^{A} X$, in a given energy domain treated by a given model, a proper evaluation of the parameter set $\mathbf{x}_{0}$ with its associated covariance matrix $M_{x}^{0}$ was performed. This can be considered as your background information $\mathrm{U}$. As a result, there is only one additional term in the cost function:

$$
\chi_{G L S}^{2}=\left(\mathbf{x}-\mathbf{x}_{m}\right)^{T} M_{x}^{-1}\left(\mathbf{x}-\mathbf{x}_{m}\right)+(\mathbf{E}-\mathbf{C}(\sigma(\mathbf{x})))^{T} M_{E}^{-1}(\mathbf{E}-\mathbf{C}(\sigma(\mathbf{x}))) .
$$

In this situation, the parameters covariances represent some most probable intervals of values for the parameters. Using an additional information coming from integral measurements will change the parameters in this most probable interval. If you have the knowledge of the parameters but no idea of the associated uncertainties. You must then do a retro-active analysis [8][10] of the parameters in order to obtain an estimated covariance matrix. Then, the problem is equivalent to previously.

Furthermore, additional problems arise when calculating $\mathrm{C}$ values ( $k_{\mathrm{eff}}$, Sodium void reactivity effect, Doppler coefficients, etc ...) as well as their derivatives $G$ : a proper interface should be made between the evaluation code, CONRAD and the neutronic tools simulating the integral experiments (ERANOS/APOLLO2, TRIPOLI-4).

Three different methods are implemented in the CONRAD framework to calculate the derivative matrix $G$ :

- the low fidelity method, where $G$ will be called $G_{l f}$,

- the coupled method, where $G$ will be called $G_{c c}$,

- the reference or exact method, where the derivative matrix will be denoted as $G_{r e f}$.

In the next chapter, only the reference method is exposed.

\subsubsection{The reference method}

Assuming that there are $N_{x}$ parameters and $N_{E}$ integral values, we will create $\left(2 N_{x}+1\right)$ evaluations from CONRAD calculations for the reference method. One of them is based on the values of the parameters set $\mathbf{x}$ and will be used to calculate $\mathbf{C}$. Then, for each parameter $x_{j}$ from $\mathbf{x}$, we will create two evaluations based respectively on the parameters vectors $\mathbf{x}_{+\delta x_{j}}=\left\{x_{0}, \ldots, x_{j}+\delta x_{j}, \ldots, x_{N_{x}}\right\}$ and $\mathbf{x}_{-\delta x_{j}}=\left\{x_{0}, \ldots, x_{j}-\delta x_{j}, \ldots, x_{N_{x}}\right\}$. With $\mathbf{x}_{+\delta x_{j}}$ and $\mathbf{x}_{-\delta x_{j}}$ we can calculate $\mathbf{C}^{+\delta x_{j}}$ and $\mathbf{C}^{-\delta x_{j}}$. Finally, the derivative matrix $G_{r e f}$ for the reference method is described as:

$$
G_{r e f}(i, j)=\frac{\partial C_{i}}{\partial x_{j}} \simeq \frac{C_{i}^{+\delta x_{j}}-C_{i}^{-\delta x_{j}}}{2 \delta x_{j}} .
$$

The major drawback of the reference method is that it is very time consuming because $\left(2 N_{x}+1\right) \cdot N_{E}$ calculations with the transport code are required. However, it is the most accurate method in the sense that all the perturbations induced by the parameters are properly propagated to the integral calculated values. Furthermore, this method can be applied with both deterministic and Monte Carlo codes.

\subsubsection{Example}

This method $[13,14]$ to use integral information has already been successfully used in the past. Another example is given in figure 3. It presents the use of PROFIL experiments on ${ }^{242} \mathrm{Pu}$ for data sssimilation onthe capture cross section. 


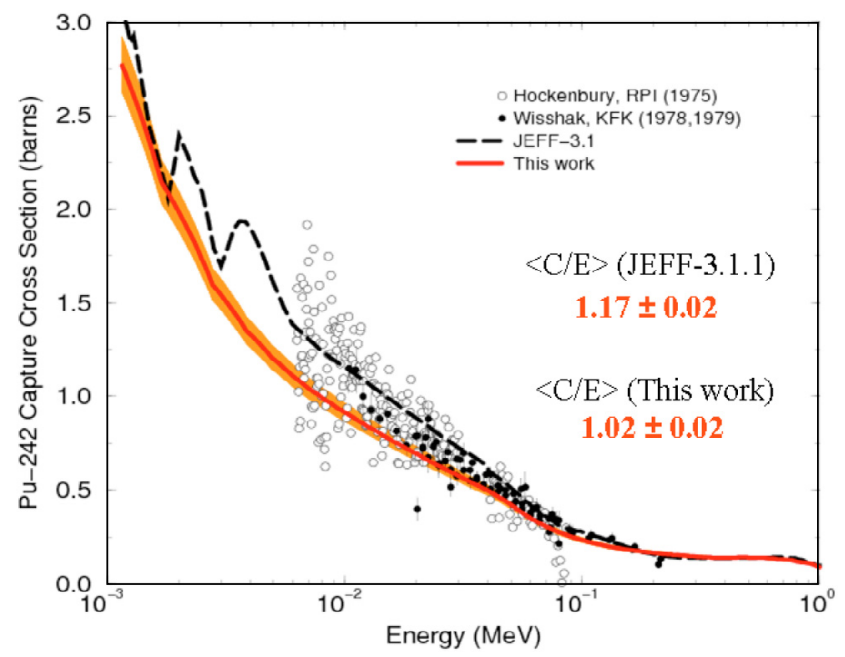

Fig. 3. ${ }^{242} \mathrm{Pu}$ capture cross section using PROFIL integral results.

\section{Conclusions}

In the CONRAD code, nuclear reaction model parameters are the vector of uncertainties. The estimation of their covariances via adjustment on microscopic data shows:

- normalisation can induced puzzling problem on the parameter estimation as well as their correlations

- solution were developped considering marginalization of experimental parameters as necessary ingredients,

- be as far as possible automatic,

- associated with validation benchmarks to avoid non-expected discrepancies.

This paper describes also a mathematical framework to add integral experiments information during the evaluation process with CONRAD. The integral experiments are seen as feedbacks for an evaluation, so they are used after microscopic experiments treatement to correct accurately the parameters.

The following conclusions can thus be made :

- use of clear integral experiment can and should be added in the evaluation process;

- best practises should be used and described (non exhaustive presentation here);

- in CEA-Cadarache an extensive use of this methodology in conjunction with proper covariances estimations is scheduled with CONRAD;

- the interaction between reactor physicists and nuclear data evaluators should:

- be imposed ....,

- append in a clear mathematical framework,

- be as far as possible automatic,

- associated with validation benchmarks to avoid non-expected discrepancies.

\section{References}

1. G. Aliberti, G. Palmiotti, M. Salvatores, T.K. Kim, T.A. Taiwo, M. Anitescu, I. Kodeli, E. Sartori, J.C. Bosq, J. Tommasi, Nuclear data sensitivity, uncertainty and target accuracy assessment for future nuclear systems, Annals of Nucl. Energy Vol 33, 700-733 (2006).

2. C. De Saint Jean et al., Proc. Int. Conf. on Nuclear Data for Science and Technology, Nice, France, 2007, (EDP Science, 2008). 
3. R. Gwin, L.W. Weston et al., Simultaneous Measurement of the Neutron Fission an Absorption Cross Sections of Plutonium-239 Over the Energy Region $0.02 \mathrm{eV}$ to $30 \mathrm{keV}$, Nuclear Science and Engineering Vol 45, 25-36 (1971).

4. Bayes, Rev. T., An Essay Toward Solving a Problem in the Doctrine of Chances, Philos. Trans. R. Soc. London 53, pp. 370-418 (1763); reprinted in Biometrika 45, pp. 293-315 (1958).

5. A. Papoulis and S.U. Pillai, Probability, Random Variables and Stochastic Processes, (McGrawHill, 2002).

6. T.M. Cover, J.A. Thomas, Elements of information theory, 2nd Edition. New York: WileyInterscience, 2006.

7. C.P. Robert and G. Casella, Monte Carlo statistical methods, Springer, 2nd edition, 2004.

8. N.M. Larson, Updated users' guide for SAMMY: multilevel R-matrix fits to neutron data using Bayes' equations, ORNL/TM-9179/7, Oak Ridge National Laboratory, Oak Ridge, TN, USA (2006).

9. C. De Saint Jean et al., Nucl. Sci. Eng. 161, 363 (2009).

10. C. De Saint Jean et al., Nucl. Sci. Eng. to be published (2010).

11. A. Gandini, M. Petilli, M. Salvatores, 1973. Nuclear data and integral measurement. Correlation for fast reactors. Statistical formulation and analysis of methods. The consistent approach. In: Symposium of Physics of fast Reactors, Tokyo.

12. P. Archier et al., ${ }^{23} \mathrm{Na}$ Evaluation with CONRAD for Fast Reactor Application, ND2010 conference (paper 1298).

13. C. De Saint Jean et al., Improved resonance shape analysis methodology in CONRAD using integral data constraints: case of ${ }^{239} \mathrm{Pu}$, Proc. Neutron Meas. Eval. - NEMEA-5, Ljubljana, Slovenia, 2008.

14. C. De Saint Jean et al., Fast Range Covariance Estimation using CONRAD, Proc. Of Wonder 2009 Workshop, Aix en provence, 2009.

15. C. De Saint Jean, B. Habert et al., in Proceedings of Nemea-5 Workshop, to be published (2010) . 